\title{
SGB Postconditioning Improves Intestinal Barrier Function by Inhibiting Autophagy in Conscious Rats Following Hemorrhagic Shock and Resuscitation
}

\section{Zhong-Hua Li}

Hebei North University

\section{Wen-Di Wang}

Hebei North University

\section{Qi Sun}

Hebei North University

\section{Ting-Jiao Suo}

Hebei North University

\section{Jia-Yi Zhai}

Hebei North University

\section{Hui-Bo Du}

Hebei North University

Zhen-Ao Zhao

Hebei North University

\section{Fu-Long Li}

Hebei North University

Chun-Yu Niu

Hebei Medical University

Zigang Zhao ( $\nabla$ zzghyl@126.com )

HeBei North University https://orcid.org/0000-0002-9434-5231

\section{Research}

Keywords: Postconditioning, Stellate ganglion blockade, Haemorrhagic shock, Autophagy, Intestinal barrier

Posted Date: March 4th, 2021

DOI: https://doi.org/10.21203/rs.3.rs-285891/v1

License: (c) (1) This work is licensed under a Creative Commons Attribution 4.0 International License. Read Full License 


\section{Abstract \\ Background}

Intestinal barrier dysfunction is the critical link of distant organ injury caused by hemorrhagic shock. The role of autophagy in ischemic intestinal injury has been paid more and more attention. Prophylactic treatment of stellate ganglion block (SGB) reduces intestinal barrier dysfunction induced by haemorrhagic shock. This study investigated the role of SGB post-processing on improved bowel barrier function and autophagy-related mechanisms of action after haemorrhagic shock.

\section{Methods}

The model of hemorrhagic shock in conscious rats was established, and the rats were treated with SGB, autophagy inhibitor 3- methyladenine (3-MA) at the time of fluid resuscitation. We detected the survival rate, intestinal loop blood flow, intestinal barrier permeability, intestinal morphology, wet/dry ratio (W/D), the intestinal barrier and autophagy marker proteins. Simultaneously, we also observed the effect of autophagy activator rapamycin (RAPA) on SGB.

\section{Results}

SGB postconditioning significantly prolonged the overall survival and enhanced survival rate of 72 hours in rats after hemorrhagic shock. SGB post-processing and 3-MA administration improved the intestinal morphology and intestinal permeability, increased the intestinal loop blood flow and expression of Z01, Occludin and Claudin-1, and decreased the intestinal W/D and LC3 and Beclin-1 expressions, expected for increased P62. Meanwhile, RAPA partially inhibited the effect of SGB on above indices in haemorrhagic shock rats.

\section{Conclusion}

SGB postconditioning alleviates the intestinal barrier dysfunction caused by haemorrhagic shock, which is related to the inhibition of excessive autophagy.

\section{Background}

Haemorrhagic shock is a critical pathological process characterized by microcirculation dysfunction and hypoperfusion, with cell damage and organ dysfunction as severe consequences ${ }^{[1]}$. Suppose the bleeding cannot be controlled in time. In that case, the hemorrhagic shock will cause excessive inflammation and oxidative stress, which will lead to multiple organ dysfunction syndrome (MODS) and threaten the lives of patients. Mesenteric vasospasm induced-ischemia during shock leads to the intestinal barrier function damage and causes the translocation of microbes and its product in the intestine to the extraintestinal 
tissues or organs, and exacerbates organ dysfunction caused by trauma and ischemia, leading to the important organs injury ${ }^{[2-5]}$. Therefore, the function of intestinal barrier plays a key role in the prognosis of haemorrhagic shock. Stellate ganglion block (SGB) is extensively used to treat ischemic pain, arrhythmia, and gastrointestinal dysfunction, ect ${ }^{[11,12]}$. It also regulates immune response and suppresses acute post-traumatic inflammation ${ }^{[13,14]}$. Studies have shown that SGB pretreatment significantly reduces the intestinal barrier damage after hemorrhagic shock, which is related to the inhibition of endoplasmic reticulum stress ${ }^{[15,16]}$. However, it is not clear whether SGB post-processing can also reduce intestinal barrier damage after hemorrhagic shock, which is pivotal to expand the clinical application of SGB.

Autophagy is a highly conservative catabolism process through intracellular lysosomes and performs an unique and the essential role to maintain cell homeostasis and survival. However, the excessive autophagy activated by strong stimulus usually causes cell damage or even death ${ }^{[6]}$. At present, the pathophysiological role of autophagy in ischemic intestinal injury is also receiving increasing attention. Previous studies showed that the appropriate activation of autophagy reduces ischemic intestinal injury. the excessive activation of autophagy aggravates the ischemic intestinal injury, while autophagy inhibition reduces intestinal mucosal barrier damage ${ }^{[7-10]}$. And then, the role of autophagy in SGB protecting the intestinal barrier is unclear. Therefore, we speculated that SGB post-processing suppressed the intestinal barrier damage caused by hemorrhagic shock by inhibiting autophagy activation. To verify this hypothesis, we saw SGB post-processing effects on survival rate, intestinal blood flow, intestinal tissue morphology, intestinal barrier function and related proteins in hemorrhagic shock rats. At the same time, we investigated the effects of 3-methyladenine (3-MA) (autophagy inhibitor) and rapamycin (RAPA) (autophagy agonist) on SGB post-processing.

\section{Methods}

\section{Experimental animal}

Eighty-eight healthy Wistar male rats were selected and purchased from SPF (Beijing) Biotechnology Co., Ltd., weighing $300 \pm 20 \mathrm{~g}$. The Animal Ethics Committee of Hebei North University approved and passed the experimental protocol.

\section{Experiment design}

A total of 52 animals were randomly split into four groups of 13, including Sham, Sham + SGB, Shock and Shock + SGB groups. These rats were used to observe and analyze the effect of SGB post-processing on the survival rate of hemorrhagic shock rats. Subsequently, another 36 rats were randomly split into Sham, Shock, Sham + SGB, Shock + SGB, Shock + 3-MA, Shock + SGB + RAPA groups, with 6 rats in each group. This part of the experiment aimed to investigate the effects of SGB post-processing on the gut barrier following haemorrhagic shock and the mechanism of autophagy. The hemorrhagic shock model in conscious rats was created in the various shock groups. The same procedure was implemented in the 
Sham and Sham + SGB groups, but without bleeding and resuscitation. One hour after shock or corresponding time point, SGB or sham SGB was administered under inhalation anaesthesia. During the recovery period, the intravenous injection of 3-MA ( $3 \mathrm{mg} / \mathrm{kg}$, Sigma, USA) and intraperitoneal injection of RAPA (10 mg/kg, Sigma, USA) were performed in the Shock + 3-MA and Shock + SGB + RAPA groups, respectively.

\section{SGB protocol}

According to the conventional method in our laboratory ${ }^{[15,16]}$, the left side stellate ganglion was blocked by injecting $0.5 \%$ ropivacaine hydrochloride (AstraZeneca AB, Sweden). After the rat spontaneously waked up, the appearance of ptosis is the sign of the success of SGB. As a control, the left stellate ganglion of the sham SGB group was given an equal amount of normal saline.

\section{Hemorrhagic shock model}

Under the induction of $2 \%$ isoflurane inhalation anaesthesia, all rats underwent surgery to establish a conscious hemorrhagic shock model. In short, All rats were implanted with polyethylene catheters through bilateral femoral arteriovenous pathways. After fixation, the catheters were sealed with heparin, and the incision was sutured. Analgesics and antibiotics were given. Heparin sodium $(1 \mathrm{~mL} / \mathrm{kg}, 500 \mathrm{U} / \mathrm{kg})$ was injected through the right femoral vein catheter for systemic anticoagulation. Through the right femoral artery catheter, the mean arterial pressure (MAP), heart rate and pulse pressure of all rats was monitored using the PowerLab biosignal acquisition system (AD Instruments, Australia). The catheter was intubated into the left femoral artery connected to a programmable single syringe pump (NE-1000, New Era Pump Systems Inc., USA) for bleeding. After the operation, the rats wake up naturally. After a stabilizing phase of 20 minutes, $40 \%$ of the body's blood volume is released within 10 minutes (whole blood volume $[\mathrm{ml}]=$ body weight $\left.[\mathrm{g}] \times 6.12 \%{ }^{[17]}\right)$. One hour after bleeding, SGB or sham SGB was given . After ten minutes of stabilization, the rats were resuscitated with fluid (mixing the drawn blood with Ringer's solution in a ratio of 1:1) through the right femoral vein for a total of 30 minutes.

\section{Survival time and survival rate}

We ligated the vascular, sutured the wound, and then kept the rats in separate cages. We recorded the survival time by video surveillance and calculated the rat's survival rate for 72 hours of observation.

\section{Intestinal loop blood flow}

Three hours after resuscitation or the corresponding time point, all rats were inhaled anaesthetized and intramuscularly injected with $10 \%$ sodium pentobarbital ( $40 \mathrm{mg} / \mathrm{kg}$, Merck, Germany) for general anaesthesia. The abdominal operation was performed at room temperature to expose the intestinal loop of about $10 \mathrm{~cm}$ upwards of the cecum, which is tiled in a circle. The laser speckle dynamic blood flow monitoring system (Pericam PSI type, Pari Medical, Sweden) was used to observe the blood flow changes in the fixed intestinal loop area, and record the average value of blood perfusion within 1 minute.

\section{Intestinal permeability}


After monitoring the intestinal loop blood flow, one segment of the ileum $(10 \mathrm{~cm}$ in length, $5 \mathrm{~cm}$ from the ileocecal area) is ligated with silk thread at both ends. And then $1 \mathrm{ml}$ of pre-prepared FITC-Dextran (FD4) solution $(10 \mathrm{mg} / \mathrm{ml}$, Sigma, USA) was injected into this section of the intestinal cavity. Don't damage mesenteric vessels during operation to prevent leakage of FITC-Dextran fluid. After 30 minutes, the portal vein blood was taken immediately, anticoagulated with heparin, and centrifuged to extract plasma (3000 $\mathrm{g}, 4^{\circ} \mathrm{C}, 10$ minutes). The fluorescence intensity of plasma (100 $\left.\mu \mathrm{l}\right)$ was measured at a SpectraMax M3 $(M D, U S A)$. Finally, according to the standard curve formula of FD4 solution $\left(y=0.011 x-0.1755, R^{2}=\right.$ $0.9992)$, the concentration of FITC-Dextran in portal vein plasma $(\mu \mathrm{g} / \mathrm{ml})$ was calculated. The intestinal tissue was then collected, fixed with $4 \%$ paraformaldehyde, and embedded in the slices. After HE staining, the distribution of FD4 was observed under a confocal microscope (Olympus, Japan).

\section{Intestinal morphology}

A section of fresh intestinal tissue (3-5 cm in length) was retained $20 \mathrm{~cm}$ from the ileocecal area and fixed in $4 \%$ paraformaldehyde fixative for 24 hours. Paraffin embedding, sectioning and HE staining were performed, and the intestinal tissue morphology difference was observed under a microscope. Images were collected using Leica microscope (DMI4000B, Germany) to monitor the intestinal mucosal structure and measure intestinal villi's height, the thickness of submucosa and muscular to analyze the degree of intestinal structural damage.

\section{Intestinal wet-dry ratio}

The intestinal tissue ( $2 \mathrm{~cm}$ in length, at $15 \mathrm{~cm}$ upward from the ileocecal end) was washed with PBS solution to wipe off the intestinal digesta. After absorbing the surface liquid, it was first weighed using a balance, which was known as the wet weight. Then the intestinal tissue was thoroughly dried in an oven at $60^{\circ} \mathrm{C}$. After drying for 72 hours, the intestinal tissue was weighed using a precise balance, which was called dry weight. Finally, the ratio of wet weight to dry weight was the wet-to-dry ratio (W/D).

\section{Western blotting analysis}

The fresh intestinal tissue of $2 \sim 3 \mathrm{~cm}\left(20 \mathrm{~cm}\right.$ from the ileocecal end) was collected and washed with $4^{\circ} \mathrm{C}$ PBS solution. Intestinal villus was collected and homogenized by conventional methods and centrifuged at $4^{\circ} \mathrm{C}$ at $12000 \mathrm{r} / \mathrm{min}$ for 10 minutes. The protein-containing supernatant is collected for further analysis. The protein sample concentration was determined with the BCA kit. The same amount of protein sample was taken and transferred to PVDF membrane (0.45um, Millipore, USA) through electrophoresis and membrane transfer process. After 1 hour of sealing with $5 \%$ skim milk, the membranes were combined with anti-LC3B (2775S, 1:1000, Cell Signaling Technology, MA, USA) and anti-P62 (55274-1-AP, 1:1000, Proteintech, Wuhan, China), Anti-Beclin-1 (ab207612, 1:1000, Abcam, Cambridge, UK), anti-Z01 (ab96587, 1:500, Abcam, Cambridge, UK), anti-Claudin-1 (28674-1-AP, 1:1000, Proteintech, Wuhan, China) and antiOccludin (ab216327, 1:1000, Abcam, Cambridge, UK). After the membrane was incubated at $4^{\circ} \mathrm{C}$ for 12 hours, it was set with the corresponding secondary antibody at $37^{\circ} \mathrm{C}$ for 1 hour. The protein bands were detected with an ultra-sensitive luminescent solution (Beijing Puli Gene Technology Co., Beijing, China). 
And then ImageQuant LAS 4000 (GE Co., Boston, USA) was used for imaging. The Quantity One software (V4.6.2, Bio-Rad Co., USA) was used to quantify the band density of the target protein.

\section{Statistical analysis}

All results were statistically analyzed by SPSS 22.0 software. Except for survival rate, all values were represented as mean \pm standard deviation (SD). Kaplane-Meier was used for survival analysis. The significance of the differences between groups was analyzed by one-way analysis of variance (ANOVA). Subsequent multiple comparisons between groups were analyzed using Tukey HSD test. When $P<0.05$, the result is regarded statistically meaning.

\section{Results}

\section{SGB post-processing increased the overall survival of rats with haemorrhagic shock}

Survival for more than 72 hours is considered long-term survival. The results showed that the rats of the Sham and Sham + SGB groups were all survived, and the survival rates were $100 \%$. The survival rate of Shock group and SGB + Shock group was compared in each time period, 24 hours (38.5\% vs $92.3 \%, \mathrm{P}<$ $0.05)$, 48 hours $(30.8 \%$ vs $61.5 \%, P<0.05)$ and 72 hours $(23.1 \%$ vs $53.8 \%, P<0.05)$. The median survival time of Shock group and SGB + Shock group was 17.5 hours and 76.9 hours. Kaplan-Meier survival analysis showed that SGB post-processing significantly improved the survival rate of rats after haemorrhagic shock, which further proved the effectiveness of SGB. The survival curve is shown in Fig. 1.

\section{Changes of MAP, heart rate and pulse pressure in rats with haemorrhagic shock}

As shown in Fig. 2a, Compared with the MAP of the Sham group, there was no striking difference in that of the Sham + SGB groups during the experiment. At different stages of blood loss and resuscitation, there was no statistically significant difference in MAP between shock, shock + SGB, shock + 3-MA, and shock + SGB + RAPA groups. But after acute hemorrhage, the MAP of the shock groups was visibly lower than that of the sham group $(P<0.05)$. Upon completion of fluid resuscitation and 3 hours thereafter, there was no striking difference in MAP among these groups $(P>0.05)$. As shown in Fig. $2 b$, there was no statistical difference in heart rate at all time points among the six groups $(P>0.05)$.

As shown in Fig. 2c, compared with the sham operation group, the pulse pressure difference in the shock group after acute bleeding was significantly reduced $(P<0.05)$. Otherwise, there was no striking difference in this index at all time points among the six groups $(P>0.05)$.

\section{SGB post-processing raised the blood flow of the intestinal loop of rats after haemorrhagic shock}


As shown in Fig. 3, the blood flow of the intestinal loop in the Shock group was significantly lower than that in the Sham group $(P<0.05)$, which was significantly increased by SGB and 3-MA treatments $(P<$ 0.05). In contrast, RAPA offset the beneficial effect of SGB on improving the blood flow of the intestinal loop of shock rats $(P<0.05)$.

\section{SGB post-processing alleviated the gut morphological damage of rats after hemorrhagic shock}

Compared with the Sham group, the Shock group's intestinal villus was thicker, shorter, and irregular, and the morphological damage was apparent. The treatment of SGB or 3-MA reduced Shock + SGB group and Shock + 3-MA group injury compared with Shock group. The intestinal tissue morphology damage in the Shock + SGB + RAPA group was more severe than that in the Shock + SGB group (Fig. 4a).

By measuring the intestinal villi height, the submucosa the thickness, and the muscle layer thickness, we found that these indicators in the Shock group were dramatically lower than those in the non-shock group $(P<0.05)$. The corresponding indices of SGB and 3-MA treatment groups were significantly higher than those of Shock group $(P<0.05)$. At the same time, the above indicators in the Shock + SGB + RAPA group were substantially lower than those in the Shock + SGB group $(P<0.05)$ (Fig. $4 b-d)$.

As shown in Fig. 4E, the W/D ratio of the Shock group was significantly higher than that in the Sham group $(P<0.05)$. SGB and 3-MA treatment reduced the intestinal W/D of rats with haemorrhagic shock ( $P$ $<0.05)$. However, the combination of RAPA offset the effect of SGB $(P<0.05)$.

\section{SGB post-processing alleviated the gut barrier of rats after haemorrhagic shock}

After perfusing with FD4 in the intestinal cavity, the distribution and quantity of FD4 in the intestinal mucosa and submucosa tissues were different among these groups (Fig. 5a). Figure $5 b$ showed that the permeability of the intestinal mucosa to FD4 in the Shock group was significantly higher compared with the Sham group $(P<0.05)$, which was decreased in the Shock + SGB and Shock + 3-MA groups $(P<0.05)$. However, the permeability of Shock + SGB + RAPA group was significantly higher than that of Shock + SGB group $(P<0.05)$. It indicated that RAPA inhibited the beneficial effect of $S G B$ on the gut mucosal barrier after acute bleeding. Western Blotting results (Fig. 5c-e) showed that the expression of tight junction proteins ZO-1, Occludin and Claudin-1 in the intestinal tissue of the Shock group was remarkably reduced compared with the Sham group $(P<0.05)$, and the intervention of SGB and 3-MA increased the expression of these proteins $(P<0.05)$. However, The RAPA treatment down-regulated the expression of the proteins mentioned above and partially offset the effect of SGB $(P<0.05)$.

\section{SGB post-processing inhibited the expression of autophagy in intestinal tissues of rats after hemorrhagic shock}


As shown in Fig. 6a-C, the expressions of LC3 (LC3-II) (a marker of autophagy activation) and Beclin-1 (a key regulator of autophagosome formation) were increased significantly after hemorrhagic shock. In contrast, autophagy SQSTM1/p62 was significantly reduced $(P<0.05)$. SGB post-processing and 3-MA administration reversed the expressions of proteins mentioned above in shock rats' intestinal tissue $(\mathrm{P}<$ 0.05). while SGB treatment had no significant effect on sham operation rats $(P>0.05)$. In addition, the autophagy activator RAPA offset the therapeutic effect of SGB post-processing $(P<0.05)$. These results confirmed that autophagy's induction role is involved in SGB post-processing, reducing the gut injury of rats after haemorrhagic shock.

\section{Discussion}

Hemorrhagic shock-induced excessive activation of the sympathetic adrenal medulla system resulted in the intestine continues state of hypoperfusion, which induces intestinal barrier damage. The current study investigated SGB post-processing effect on intestinal barrier function following haemorrhagic shock and found that SGB post-processing significantly improved the long-term survival rate and intestinal barrier function, which was related to the inhibition of autophagy activation.

The stellate ganglion belongs to the sympathetic ganglion, and the electrical activity of its neurons participates in the regulation of the autonomic nervous system ${ }^{[19]}$. The beneficial effects of SGB are mostly attributed to the inhibition of sympathetic hyperexcitability ${ }^{[18]}$. The previous study showed that SGB could promote local blood circulation, reduce inflammation, and regulate the nerve-endocrineimmune system's function, which plays a positive role in maintaining the environment's homeostasis ${ }^{[20]}$. This study found that SGB post-processing dramatically prolonged the survival time and enhanced the survival rate of 72 hours in rats after hemorrhagic shock, suggesting that SGB post-processing is of great significance to the overall intervention hemorrhagic shock.

In severe trauma or shock, the intestine is one of the organs with the earliest hypo-perfusion. Studies have reported that when blood loss occurs but no systemic symptoms or vital signs occur, the small intestine and colon blood flow has decreased by $50 \%{ }^{[21]}$. However, after resuscitation, the intestinal blood perfusion is restored at the latest ${ }^{[22]}$, making the mesenteric microcirculation in a state of continuous ischemia for a long time during shock resuscitation. In the experiment, we observed the loop blood flow of rats after hemorrhagic shock and found that the intestinal perfusion in the Shock group was significantly reduced, but the intervention of SGB significantly increased the intestinal blood flow in the Shock group. A recent systematic review and meta-analysis of the effect of SGB on the recovery of gastrointestinal function in patients undergoing general anesthesia confirmed the effect of SGB in promoting gastrointestinal recovery after traumatic surgery, which was related to gastrointestinal blood vessels telangiectasis and gastrointestinal motility improvement through the blockage of sympathetic nerves that innervate the gastrointestinal system ${ }^{[23]}$. The present result suggests that SGB postprocessing improves the intestinal blood supply after hemorrhagic shock, which is beneficial for reducing intestinal damage. 
When intestinal ischemia and hypoxia occur, gathering many acidic metabolites in the local area directly causes cell metabolism dysfunction and tissue damage and reduces intestinal mucosal epithelial oedema, intraepithelial cell connections rupture, and even cell necrosis. Loss of the top of the mucosa's villi or full-thickness increases intestinal permeability and destroys the intestinal mucosal barrier ${ }^{[24]}$. Our current work found that SGB postconditioning improved the histomorphology and oedema of intestinal mucosa after hemorrhagic shock. Since intestinal mucosal barrier dysfunction is closely related to increased intestinal permeability ${ }^{[25]}$, we also tested the plasma concentration of FD4 and the distribution of FD4 in intestinal mucosa and submucosal tissues, and the results showed that SGB postconditioning significantly reduced intestinal mucosal permeability after hemorrhagic shock. These pieces of evidence demonstrated that SGB post-processing relieved intestinal barrier dysfunction after the hemorrhagic shock to a certain extent, which is consistent with the role of SGB pre-treatment ${ }^{[15,16]}$.

The tight junction $(\mathrm{TJ})$ is mainly a composite structure composed of the transmembrane protein family (occludin and claudin protein) and the peri-membrane protein family (ZO protein) ${ }^{[26,27]}$, which is the basis for the integrity of the intestinal mucosal barrier structure. The ZO protein connects the Claudin protein, the occludin protein and the actin backbone system together to form a stable tight junction structure ${ }^{[28]}$. Many studies have confirmed that the expression of tight junction proteins ZO-1, Claudin-1 and occludin are good indicators of intestinal barrier function and permeability function ${ }^{[29,30]}$. This study showed that SGB post-processing significantly up-regulated the expressions of ZO-1, Claudin-1 and occludin in shock rats, indicating that SGB post-processing further reduced intestinal barrier dysfunction after hemorrhagic shock.

In generally, autophagy at the physiological level is believed to help maintain the balance between tolerance and defense in the intestine, but excessive autophagy activation has the opposite effect ${ }^{[31]}$. The mechanism of ischemic intestinal injury may be related to trigger a series of complex cascade reactions, such as ROS burst, mitochondrial calcium overload and neutrophil infiltration, which are all effective activating factors of autophagy and lead to overexpression of autophagy ${ }^{[32]}$. Our research showed that hemorrhagic shock and resuscitation enhanced the activation of autophagy. On the contrary, SGB postprocessing down-regulates autophagy expression in the intestine, which is consistent with the tendency of SGB repairing the intestinal barrier.

On the other hand, we used autophagy inhibitor (3-MA) and activator (RAPA), combined with other intestinal injury evidence, to analyze the effect of autophagy on the intestinal damage caused by haemorrhagic shock and the correlation between SGB post-processing and autophagy. 3-MA has an observably inhibitory effect on the activity of phosphatidylinositol 3-kinase (PI3K), which is an essential component for the recruitment and formation of autophagic vesicles. Therefore, 3-MA blocks the autophagy process and autophagy formation at an early stage in mammals, and has been widely used in the study of autophagy pathophysiology ${ }^{[33-35]}$. RAPA is a macrolide compound produced by streptomyces hygroscopicus. It targets mammalian target of rapamycin (mTOR) and activates autophagy by inhibiting mTOR phosphorylation ${ }^{[36]}$. When 3-MA was used to intervene in Shock group, we 
found that autophagy was inhibited. Simultaneously, 3-MA significantly alleviated intestinal mucosal tissue morphology injury and increased intestinal blood perfusion and reduced intestinal mucosal permeability. These findings indicate that autophagy inhibition by 3-MA is beneficial to lessen the intestinal barrier damage caused by hemorrhagic shock and resuscitation, which is consistent with the effect of SGB post-processing treatment. However, when RAPA was used in the Shock + SGB group, the expression of autophagy was significantly increased. At the same time, the treatment of RAPA significantly offset the beneficial effect of SGB post-processing treatment on the protection of the intestinal barrier. Therefore, it is reasonable to think that SGB post-processing alleviates intestinal barrier dysfunction by inhibiting autophagy. However, our study did not detect the expressions of PI3K and mTOR; then, the role of autophagy-related signaling pathway should be certified in the future.

Since the occurrence of traumatic or hemorrhagic shock is often unpredictable, preventive treatment is impossible. Therefore, we chose to study the therapeutic effect of SGB after hemorrhagic shock in the conscious rats, which is more in line with the actual situation of clinical emergencies. We first verified the effect and mechanism of SGB post-processing on the intestinal injury following hemorrhagic shock. In combination with previous studies on SGB pre-treatment results ${ }^{[15,16]}$, SGB pre-treatment or posttreatment effectively reduces the intestinal barrier damage after hemorrhagic shock. Still, neither affects blood pressure during acute bleeding.

\section{Conclusion}

SGB post-processing dramatically improves the survival rate and intestinal barrier function after haemorrhagic shock, which is carried out by inhibiting autophagy. The more extensive application of SGB and the anti-shock mechanism will be the focus in the future.

\section{Abbreviations}

SGB: Stellate ganglion block; W/D: Wet/dry ratio; RAPA: Rapamycin; 3-MA: 3- methyladenine; MODS: Multiple organ dysfunction syndrome; MAP: Mean arterial pressure; FD4: FITC-Dextran; ANOVA: Analysis of variance; PI3K: Phosphatidylinositol 3-kinase; mTOR: Mammalian target of rapamycin.

\section{Declarations}

\section{Acknowledgements}

The authors thank all students and laboratory technicians for their assistance.

\section{Authors' contributions}

ZHL performed the majority of animal experiment and laboratory work. WDW, QS, TJS, JYZ, HBD and ZAZ participated in some experimental work. ZHL acquired and analyzed the data. ZHL wrote the manuscript. 
CYN and ZGZ were involved in the conception and design of the study, data interpretation, and critically revised the manuscript. All authors read and approved the final manuscript.

\section{Funding}

This study was supported by the National Natural Science Foundation of China (No. 81770492).

\section{Availability of data and materials}

All data generated or analyzed during this study are included in this published

article.

\section{Ethics approval and consent to participate}

The experimental protocol was approved by the Animal Ethics Committee of Hebei North University.

\section{Competing interests}

The authors declare that they have no competing interests.

\section{References}

1. Peiniger S, Paffrath T, Mutschler M, et al. The trauma patient in hemorrhagic shock: how is the Cpriority addressed between emergency and ICU admission?[J]. Scand J Trauma Resusc Emerg Med, 2012, 20: 78.

2. Sonnier DI, Makley AT, Friend LA, et al. Hemorrhagic shock induces a proinflammatory milieu in the gut lumen[J]. J Surg Res, 2011, 170(2): 272-279.

3. Deng Z, Zeng H, Liang S, et al. [Dynamic effects of adjunct peritoneal resuscitation on the early intestinal injury of rats after hemorrhagic shock][J]. Zhonghua Wei Zhong Bing Ji Jiu Yi Xue, 2015, 27(1): 59-63.

4. Wells JM, Brummer RJ, Derrien M, et al. Homeostasis of the gut barrier and potential biomarkers[J]. Am J Physiol Gastrointest Liver Physiol, 2017, 312(3): G171-g193.

5. Haq S, Grondin J, Banskota S, et al. Autophagy: roles in intestinal mucosal homeostasis and inflammation[J]. J Biomed Sci, 2019, 26(1): 19.

6. Shiomi M, Miyamae M, Takemura G, et al. Induction of autophagy restores the loss of sevoflurane cardiac preconditioning seen with prolonged ischemic insult[J]. Eur J Pharmacol, 2014, 724: 58-66.

7. Li B, Yao X, Luo Y, et al. Inhibition of autophagy attenuated intestinal injury After Intestinal I/R via mTOR Signaling[J]. J Surg Res, 2019, 243: 363-370.

8. Song G, Liu D, Geng X, et al. Bone marrow-derived mesenchymal stem cells alleviate severe acute pancreatitis-induced multiple-organ injury in rats via suppression of autophagy[J]. Exp Cell Res, 2019, 385(2): 111674. 
9. Yamoto M, Lee C, Chusilp S, et al. The role of autophagy in intestinal epithelial injury[J]. Pediatr Surg Int, 2019, 35(12): 1389-1394.

10. Zeng Z, Liu HM, Zhang YY, et al. Aggravated intestinal ischemia-reperfusion injury is associated with activated mitochondrial autophagy in a mouse model of diabetes[J]. Mol Med Rep, 2020, 22(3): 1892-1900.

11. Gunduz $\mathrm{OH}$, Kenis-Coskun O. Ganglion blocks as a treatment of pain: current perspectives[J]. J Pain Res, 2017, 10: 2815-2826.

12. Zhao HY, Yang GT, Sun NN, et al. Efficacy and safety of stellate ganglion block in chronic ulcerative colitis[J]. World J Gastroenterol, 2017, 23(3): 533-539.

13. Yokoyama M, Nakatsuka $\mathrm{H}$, Itano $\mathrm{Y}$, et al. Stellate ganglion block modifies the distribution of lymphocyte subsets and natural-killer cell activity[J]. Anesthesiology, 2000, 92(1): 109-115.

14. Yang X, Shi Z, Li X, et al. Impacts of stellate ganglion block on plasma NF-KB and inflammatory factors of TBI patients[J]. Int J Clin Exp Med, 2015, 8(9): 15630-15638.

15. Zhang J, Lin XR, Zhang YP, et al. Blockade of stellate ganglion remediates hemorrhagic shockinduced intestinal barrier dysfunction[J]. J Surg Res, 2019, 244: 69-76.

16. Yin M, Li ZH, Wang C, et al. Stellate ganglion blockade repairs intestinal mucosal barrier through suppression of endoplasmic reticulum stress following hemorrhagic shock[J]. Int J Med Sci, 2020, 17(14): 2147-2154.

17. Hu $X$, Zhang $M$, Duan $X$, et al. Sevoflurane postconditioning improves the spatial learning and memory impairments induced by hemorrhagic shock and resuscitation through suppressing IRE1 acaspase-12-mediated endoplasmic reticulum stress pathway[J]. Neurosci Lett, 2018, 685: 160-166.

18. Chen $Y Q, X i e Y Y$, Wang $B$, et al. Effect of stellate ganglion block on hemodynamics and stress responses during CO(2)-pneumoperitoneum in elderly patients[J]. J Clin Anesth, 2017, 37: 149-153.

19. Cheng LJ, Li GP, Li J, et al. Effects of fluvastatin on characteristics of stellate ganglion neurons in a rabbit model of myocardial ischemia[J]. Chin Med J (Engl), 2016, 129(5): 549-556.

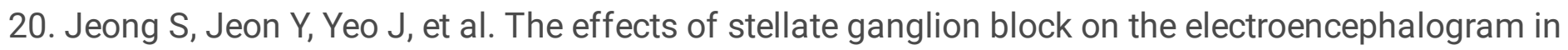
rats[J]. J Anesth, 2014, 28(4): 601-605.

21. Jeschke MG, Herndon DN, Finnerty CC, et al. The effect of growth hormone on gut mucosal homeostasis and cellular mediators after severe trauma[J]. J Surg Res, 2005, 127(2): 183-189.

22. Secchi A, Ortanderl JM, Schmidt W, et al. Effect of endotoxemia on hepatic portal and sinusoidal blood flow in rats[J]. J Surg Res, 2000, 89(1): 26-30.

23. Wen $B$, Wang $Y$, Zhang $C$, et al. Effect of stellate ganglion block on postoperative recovery of gastrointestinal function in patients undergoing surgery with general anaesthesia: a metaanalysis[J]. BMC Surg, 2020, 20(1): 284.

24. Higuchi $S$, Wu R, Zhou M, et al. Gut hyperpermiability after ischemia and reperfusion: attenuation with adrenomedullin and its binding protein treatment[J]. Int J Clin Exp Pathol, 2008, 1(5): 409-418. 
25. Salim SY, Söderholm JD. Importance of disrupted intestinal barrier in inflammatory bowel diseases[J]. Inflamm Bowel Dis, 2011, 17(1): 362-381.

26. Harhaj NS, Antonetti DA. Regulation of tight junctions and loss of barrier function in pathophysiology[J]. Int J Biochem Cell Biol, 2004, 36(7): 1206-1237.

27. Blikslager AT, Moeser AJ, Gookin JL, et al. Restoration of barrier function in injured intestinal mucosa[J]. Physiol Rev, 2007, 87(2): 545-564.

28. Smalley KS, Brafford P, Haass NK, et al. Up-regulated expression of zonula occludens protein-1 in human melanoma associates with $\mathrm{N}$-cadherin and contributes to invasion and adhesion[J]. Am J Pathol, 2005, 166(5): 1541-1554.

29. Costantini TW, Loomis WH, Putnam JG, et al. Burn-induced gut barrier injury is attenuated by phosphodiesterase inhibition: effects on tight junction structural proteins[J]. Shock, 2009, 31(4): 416422.

30. Thuijls G, de Haan JJ, Derikx JP, et al. Intestinal cytoskeleton degradation precedes tight junction loss following hemorrhagic shock[J]. Shock, 2009, 31(2): 164-169.

31. Randall-Demllo S, Chieppa M, and Eri R. Intestinal epithelium and autophagy: partners in gut homeostasis[J]. Front Immunol, 2013, 4: 301.

32. Vollmar B, Menger MD. Intestinal ischemia/reperfusion: microcirculatory pathology and functional consequences[J]. Langenbecks Arch Surg, 2011, 396(1): 13-29.

33. Suzuki C, Isaka $Y$, Takabatake $Y$, et al. Participation of autophagy in renal ischemia/reperfusion injury[J]. Biochem Biophys Res Commun, 2008, 368(1): 100-106.

34. Gotoh K, Lu Z, Morita M, et al. Participation of autophagy in the initiation of graft dysfunction after rat liver transplantation[J]. Autophagy, 2009, 5(3): 351-360.

35. Jiang M, Liu K, Luo J, et al. Autophagy is a renoprotective mechanism during in vitro hypoxia and in vivo ischemia-reperfusion injury[J]. Am J Pathol, 2010, 176(3): 1181-1192.

36. Noack M, Richter-Landsberg C. Activation of autophagy by rapamycin does not protect oligodendrocytes against protein aggregate formation and cell death induced by proteasomal inhibition[J]. J Mol Neurosci, 2015, 55(1): 99-108.

\section{Figures}




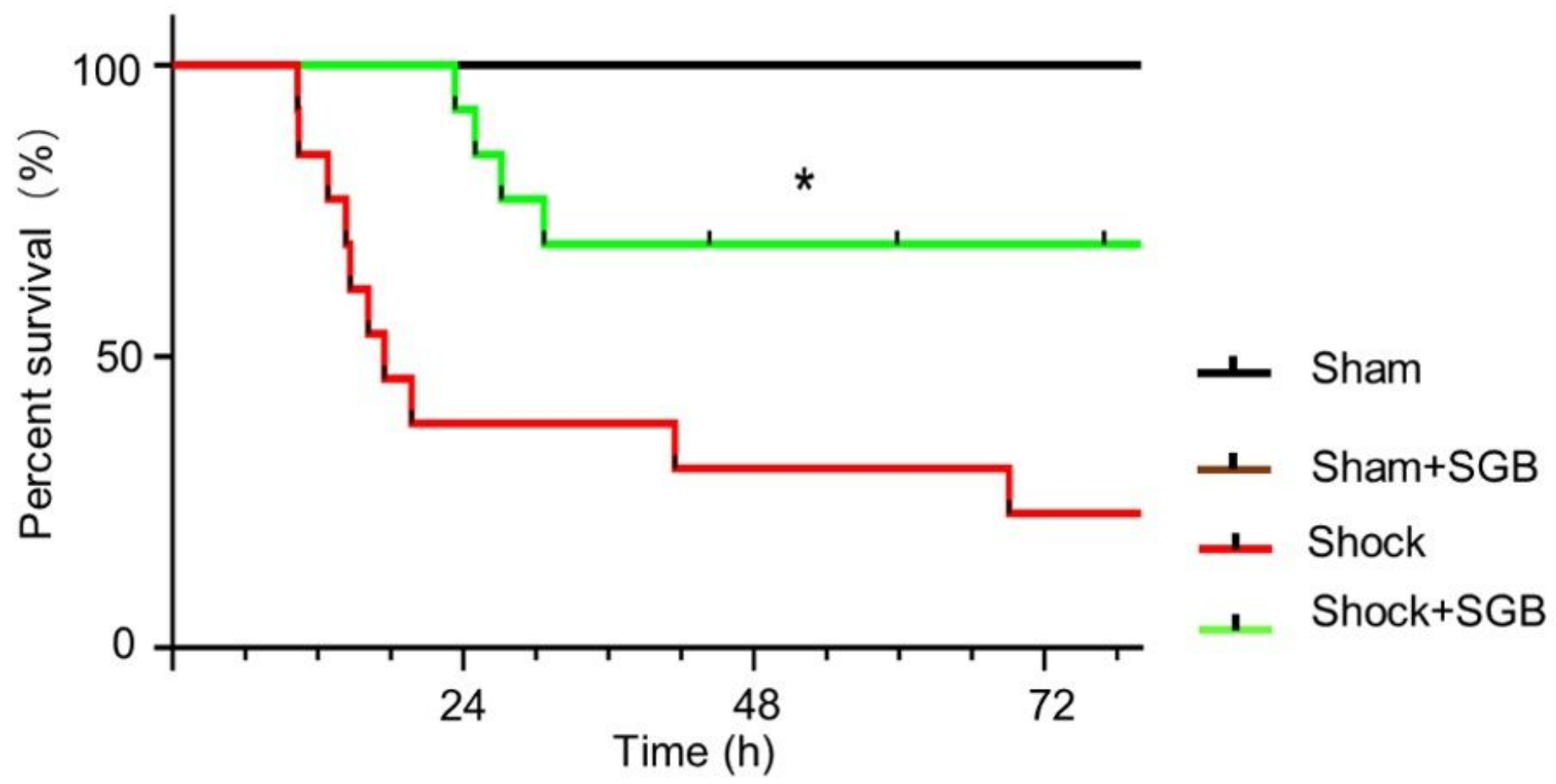

Figure 1

Stellate ganglion block (SGB) post-treatment prolonged the survival time and enhanced the survival rate of $72 \mathrm{~h}$ in conscious rats after hemorrhagic shock. $\mathrm{n}=13$ for each group. ${ }^{*} \mathrm{P}<0.05 \mathrm{vs}$. the shock group. 
a $\square$ Sham $\square$ Shock $\square$ Sham+SGB $\square$ Shock+SGB $\square$ Shock+3MA $\square$ Shock+SGB+RAPA

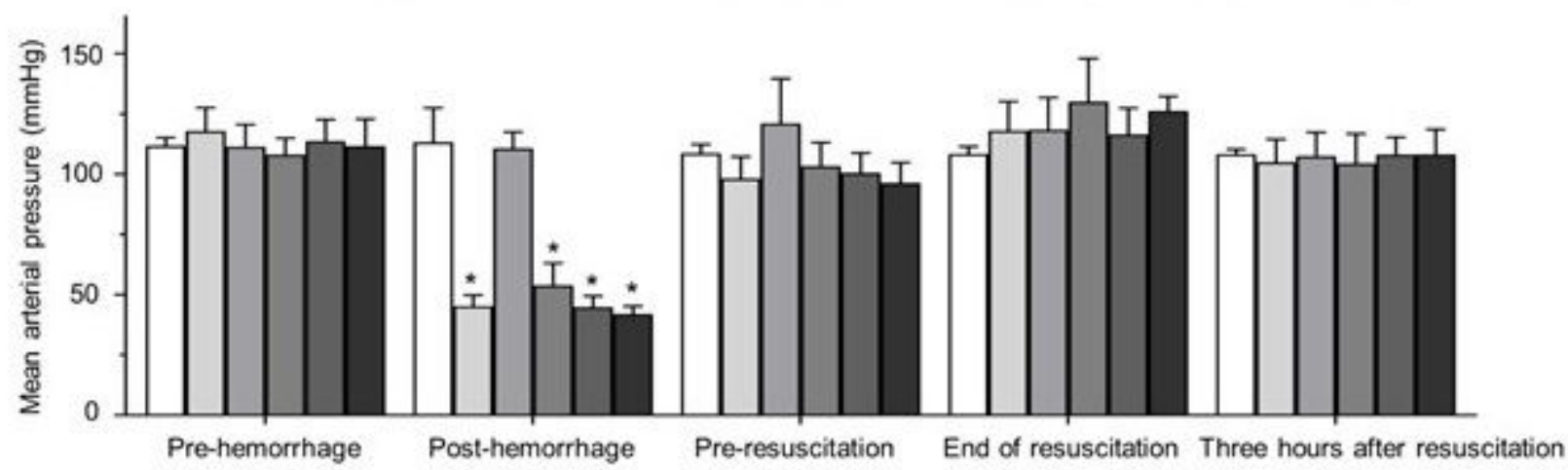

b

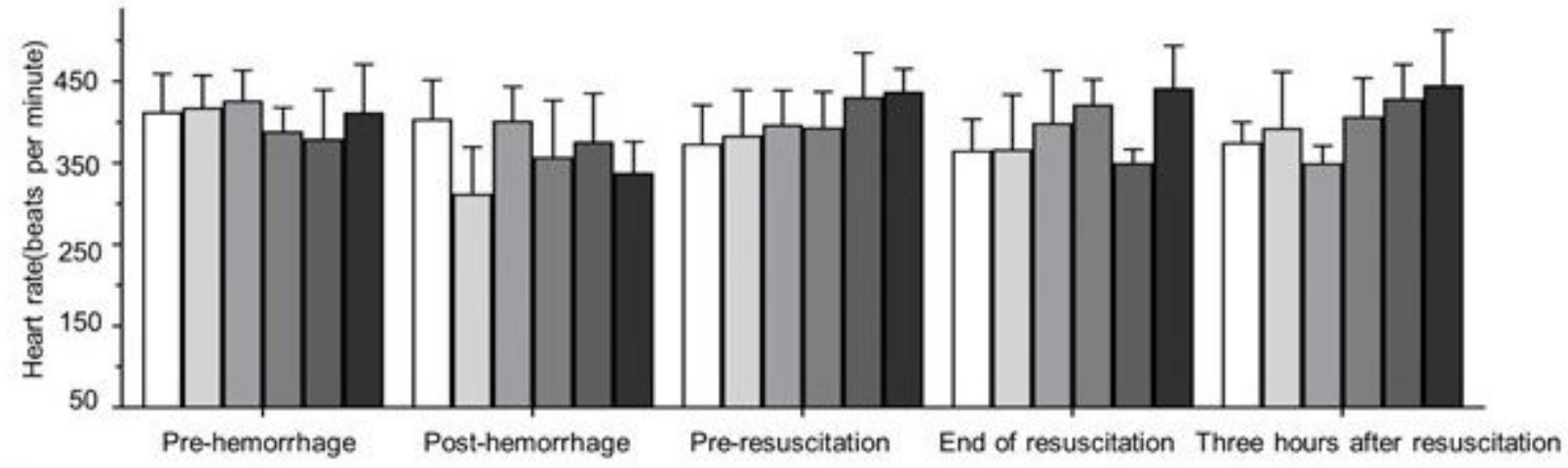

C

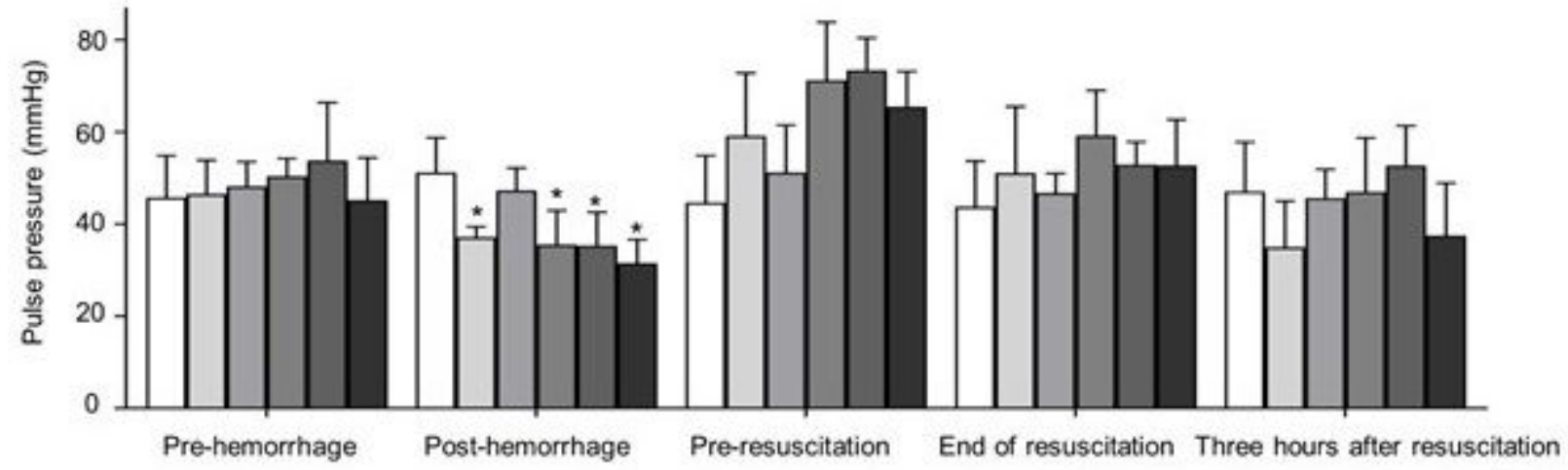

Figure 2

Changes of MAP, heart rate and pulse pressure in conscious rats after hemorrhagic shock. Data are presented as mean \pm standard, with $n=5-6$ for each group. * $P<0.05$ vs. the Sham group. 

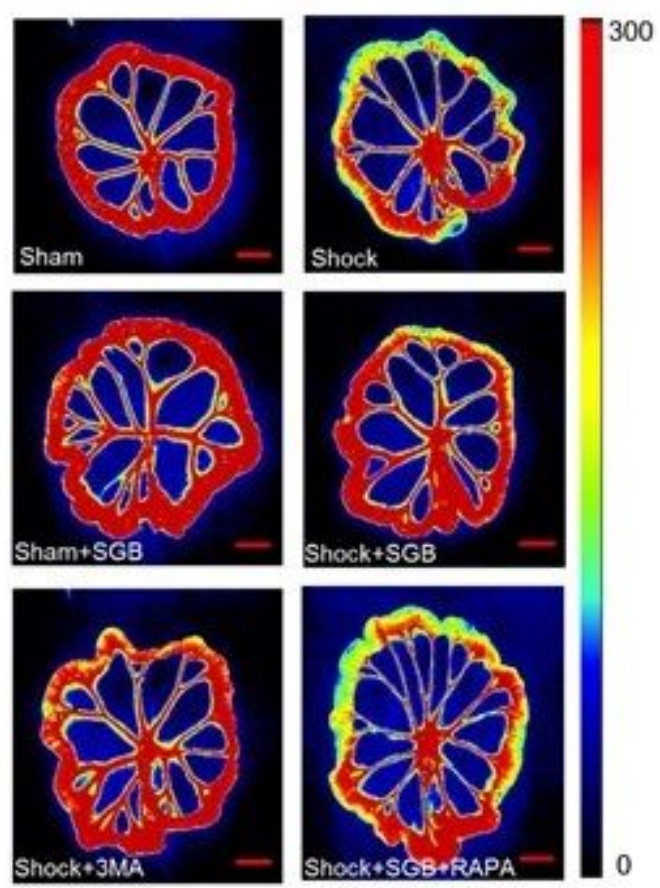

b

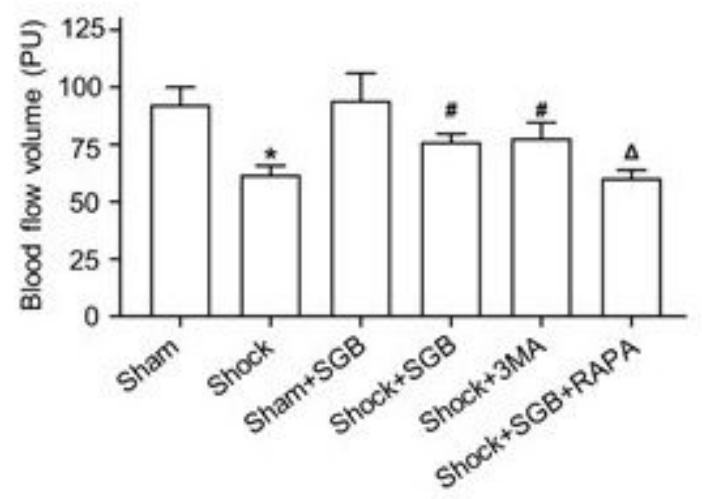

Figure 3

Stellate ganglion block (SGB) post-treatment enhanced the intestinal loop blood flow through inhibition of autophagy in conscious rats after hemorrhagic shock. a. Images of original recording $(B a r=1 \mathrm{~cm})$. b. Results of intestinal loop blood flow. Data are presented as mean \pm standard, with $n=3$ for each group. * $\mathrm{P}<0.05$ vs. the Sham group; \#P<0.01 vs. the Shock group; $\triangle \mathrm{P}<0.05$ vs. the Shock+ SGB group. 3-MA: 3methyladenine, an autophagy inhibitor; PARA: rapamycin, an autophagy activator. 
a
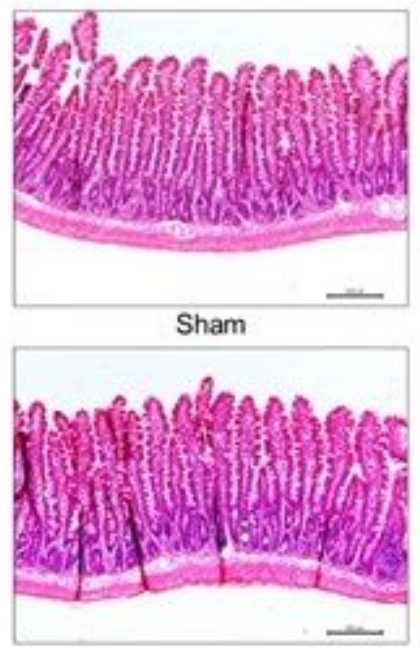

Sham+SGB

b

d
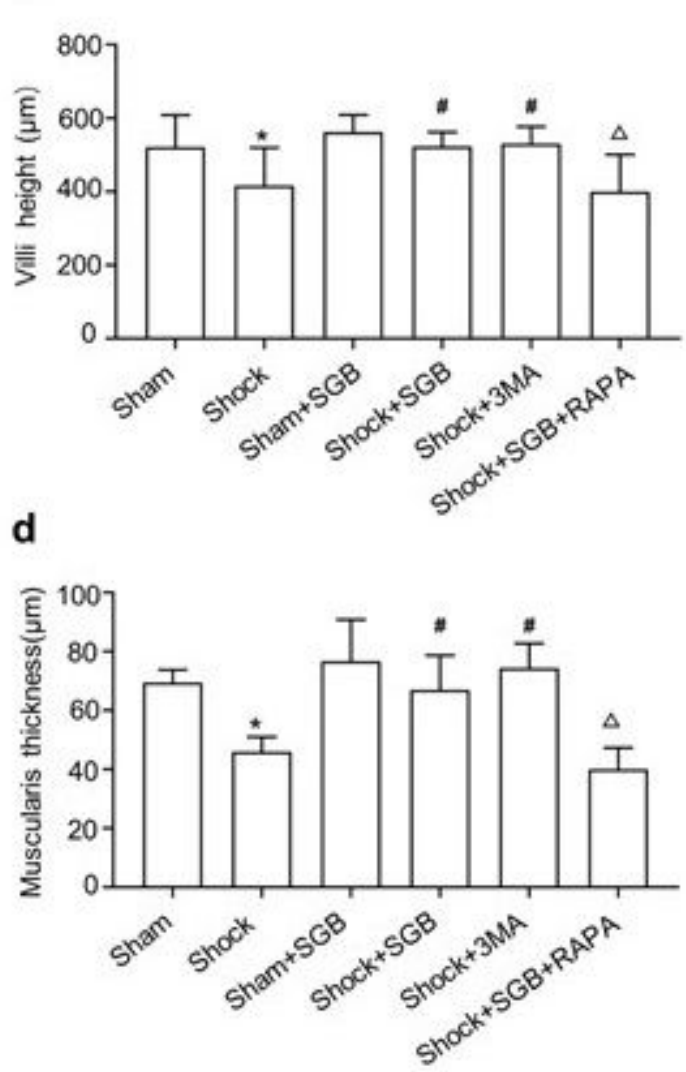

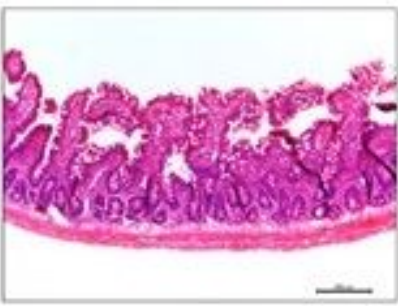

Shock

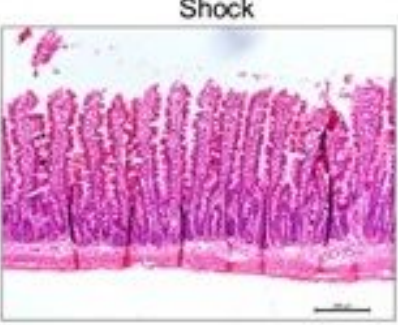

Shock+SGB

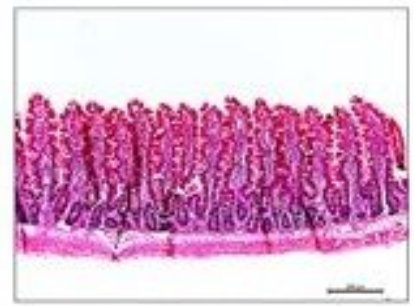

Shock+3MA

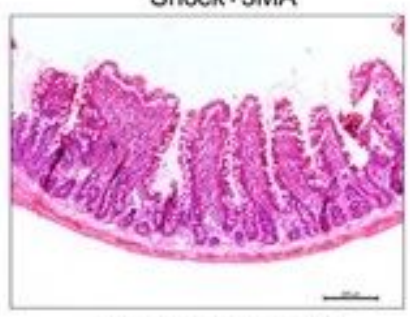

Shock+SGB+RAPA

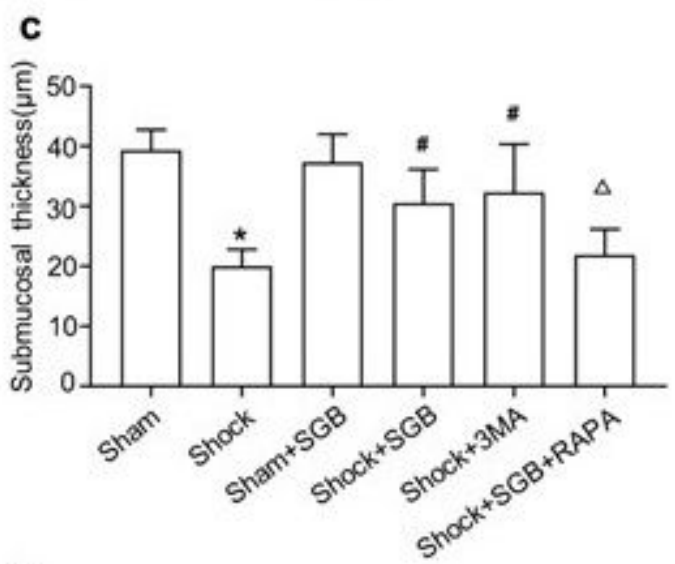

e

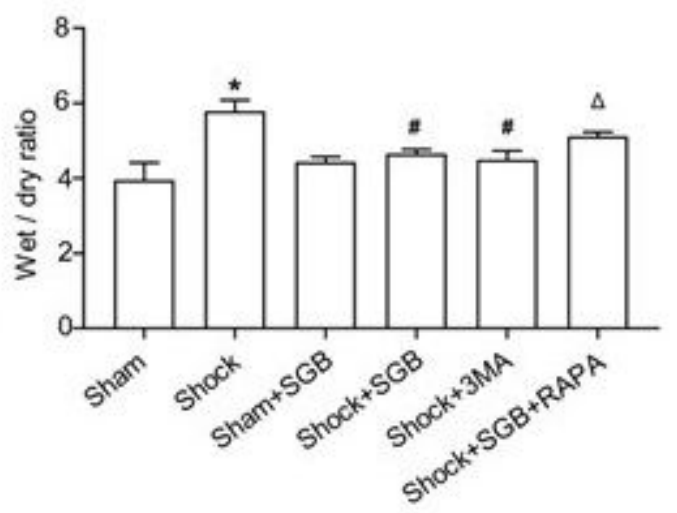

Figure 4

Stellate ganglion block (SGB) post-treatment alleviated hemorrhagic shock induced-intestinal injury through inhibition of autophagy in conscious rats. a. Characteristic images of intestinal histopathology (HE staining, Bar $=200 \mu \mathrm{m}$ ); b. Height of intestinal villus; c. Submucosal thickness; d. Muscularis thickness; e. Wet/dry ratio (W/D) of intestines. Data are presented as mean \pm standard, with $n=3$ for each group. ${ }^{*} \mathrm{P}<0.05$ vs. the Sham group, $\# \mathrm{P}<0.05$ vs. the Shock group, $\triangle P<0.05$ vs. the Shock+ SGB group. 3-MA: 3-methyladenine, an autophagy inhibitor; PARA: rapamycin, an autophagy activator. 
a
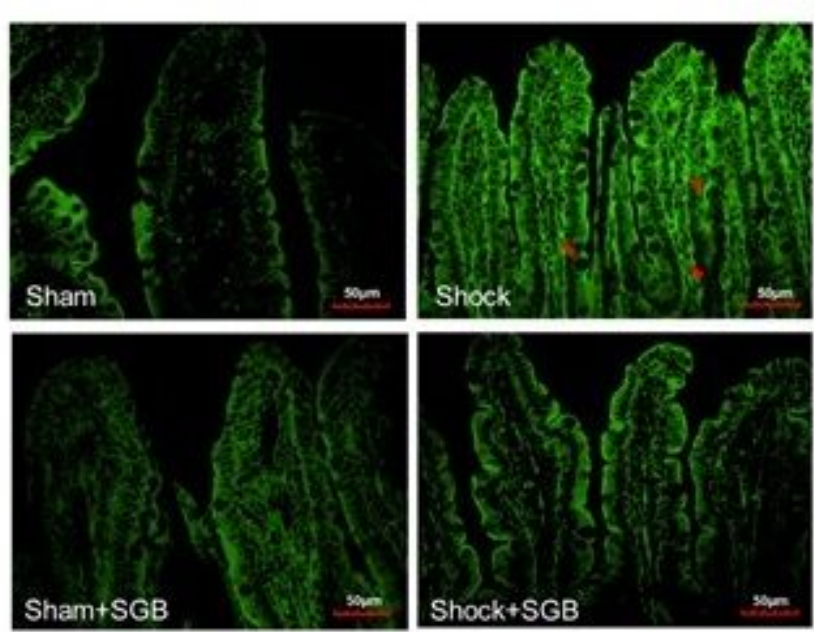

Shock+3M

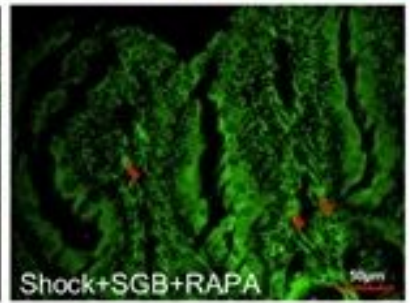

b

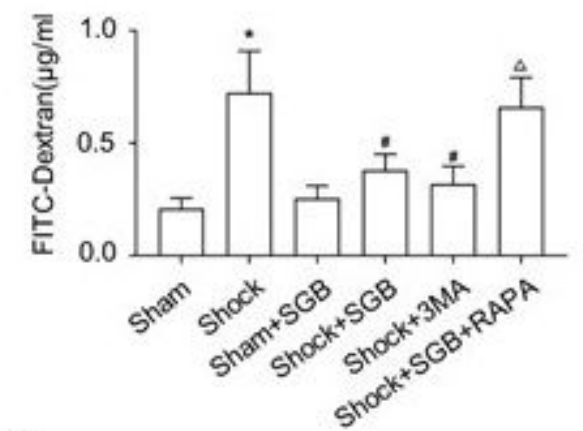

d
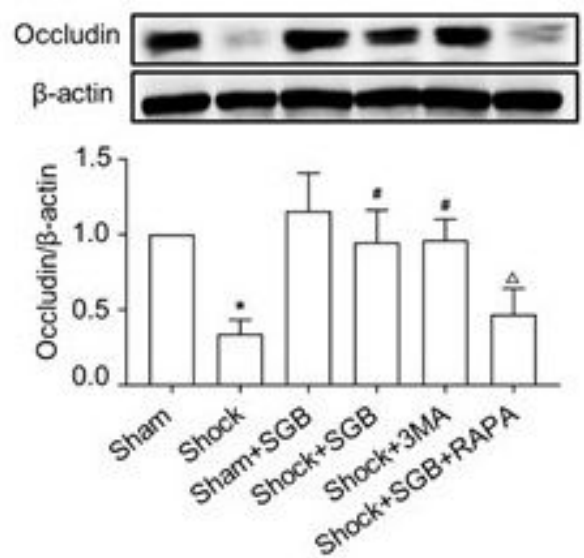
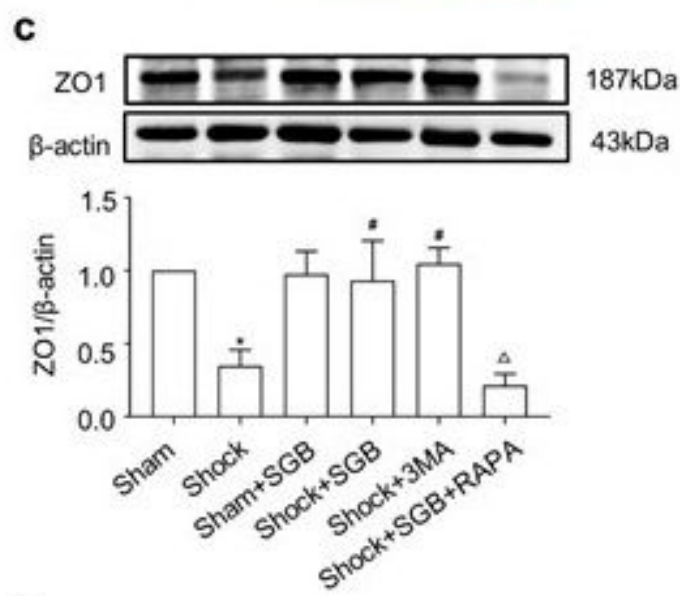

e
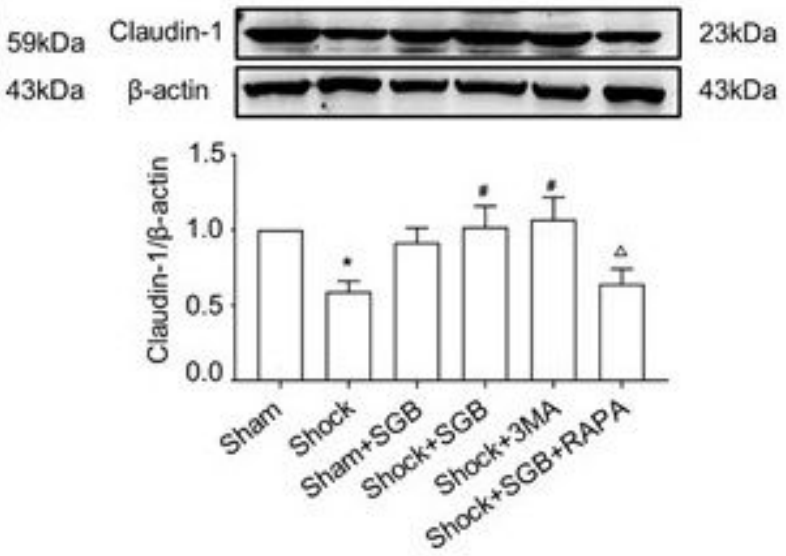

\section{Figure 5}

Stellate ganglion block (SGB) post-treatment reduced intestinal barrier damage through inhibition of autophagy in conscious rats with hemorrhagic shock. a. Image of fluorescence distribution in intestinal mucosa of rats in different groups following hemorrhagic shock. b. Intestinal clearance of fluorescein isothiocyanate-dextran (FD4) in rats, with $n=6$ for each group. c-e: SGB increased the expression of ZO-1, Occludin and Claudin- 1 in the intestinal tissue of rats with hemorrhagic shock, with $n=3$ for each group. 
Data are presented as mean \pm standard. ${ }^{*} \mathrm{P}<0.05$ vs. the Sham group, \#P<0.05 vs. the Shock group, $\triangle \mathrm{P}$ $<0.05$ vs. the Shock +SGB group. 3-MA: 3-methyladenine, an autophagy inhibitor; PARA: rapamycin, an autophagy activator.
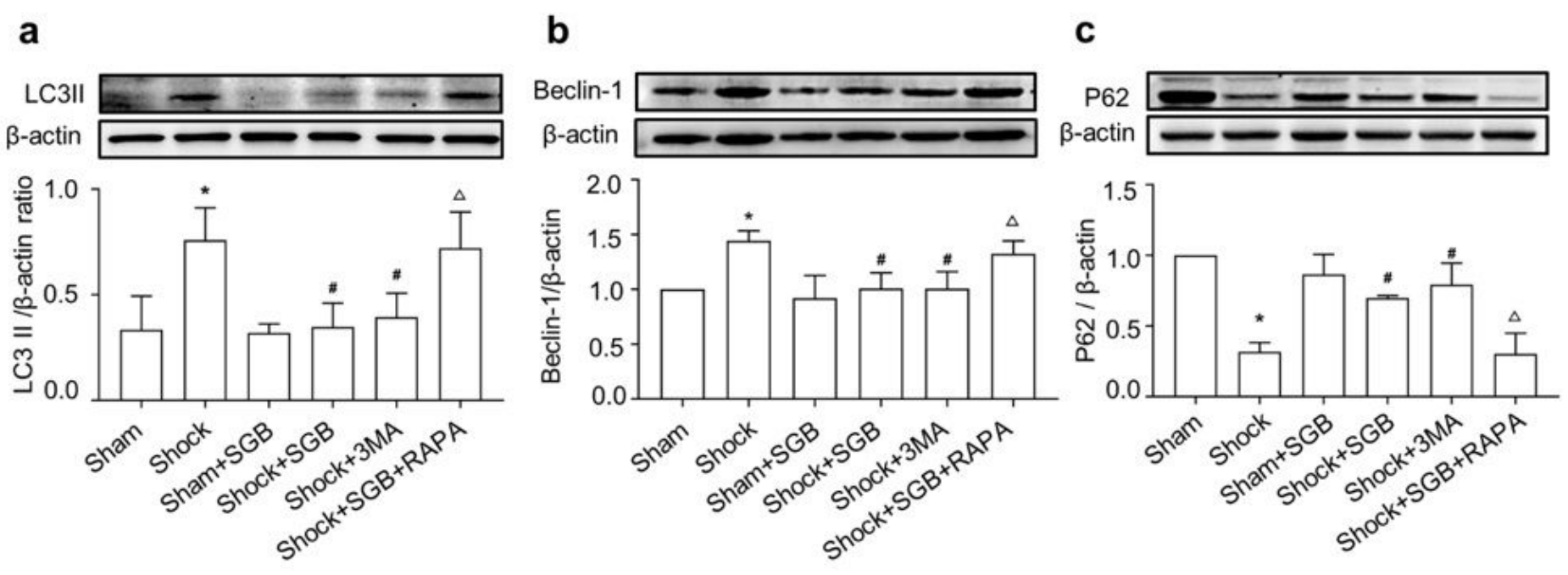

\section{Figure 6}

Stellate ganglion block (SGB) post-treatment inhibited the expressions of LC3, Beclin-1 and increased the expression of p62 of the intestinal tissue of conscious rats with hemorrhagic shock. Data are presented as mean \pm standard, with $n=3$ for each group. ${ }^{*}<<0.05$ vs. the Sham group, $\# P<0.05$ vs. the Shock group, $\Delta \mathrm{P}<0.05$ vs. the Shock + SGB group. 3-MA: 3-methyladenine, an autophagy inhibitor; PARA: rapamycin, an autophagy activator. 